\title{
Impact of Weather Parameters on Yield of Kharif Sunflower (Helianthus annuus L.) under Different Growing Environments in Scarcity Zone of Maharashtra
}

\author{
V. M. Londhe, S. G. Birajdar*, V. T. Jadhav, J. D. Jadhav and V. M. Amrutsagar
}

Zonal Agriculture Research Station, Solapur, India

*Corresponding author

\section{A B S T R A C T}

\begin{tabular}{|l|}
\hline Ke y w o r d s \\
Kharif sunflower, \\
$\begin{array}{l}\text { Sowing windows, } \\
\text { Yield attributes, } \\
\text { Cultivar }\end{array}$ \\
\hline Article Info \\
\hline $\begin{array}{l}\text { Accepted: } \\
\text { 12 August } 2020 \\
\text { Available Online: } \\
\text { 10 September 2020 }\end{array}$ \\
\hline
\end{tabular}

Present investigation entitled "Impact of weather parameters on yield of kharif sunflower (Helianthus аппииs L.) Under different growing environments in scarcity zone of Maharashtra." was carried out during 2013-17 at Zonal Agricultural Research Station, Solapur, Maharashtra State (India). The experiment was conducted in split plot design with three replications. Nine treatment combinations were formed considering different cultivars viz., $\mathrm{V}_{1}$ Bhanu, $\mathrm{V}_{2}$ MSFH-1 and $\mathrm{V}_{3}$ Phule Bhaskar and sowing windows viz., $\left(\mathrm{S}_{1}\right)$ $2^{\text {nd }}$ fortnight of June ( $\left.25^{\text {th }}-J u n e\right),\left(S_{2}\right)-2^{\text {nd }}$ fortnight of July ( $\left.27^{\text {th }}-J u l y\right) S_{3}-2$ nd fortnight of August (24 ${ }^{\text {th }}$-August). Among the three pearl millet sowing window crop sown in second fortnight of July (S2) produced significantly highest grain yield (1708.2 $\left.\mathrm{kg} \mathrm{ha}^{-1}\right)$ and total monetary returns $\left(56320 \mathrm{~kg} \mathrm{ha}^{-1}\right),($ CUM $)(320 \mathrm{~mm})$, (MUE) $\left(5.3 \mathrm{Kg} \mathrm{ha}^{-1} \mathrm{~mm}\right),($ GDD) $\left(1042^{0}\right.$ days $)$, total dry matter $\left(400.8 \mathrm{~g} \mathrm{~m}^{-2}\right)$, LAI and RUE July $\left(1.31 \& 1.63 \mathrm{~g} \mathrm{MJ}^{-1}\right)$ than other dates of sowing. Among the genotypes Phule Bhaskar produced significantly higher grain yield (1446.8 $\left.\mathrm{kg} \mathrm{ha}^{-1}\right)$, total monetary returns (Rs. $\left.47590 \mathrm{ha}^{-1}\right)$, CUM $(293.1 \mathrm{~mm}$ ), MUE (4.8 $\mathrm{Kg} \mathrm{ha}^{-1} \mathrm{~mm}$ ), mean number of days to attain physiological stages (92 days), $\operatorname{GDD}\left(846^{0}\right.$ days $)$, mean total dry matter $\left(404.1 \mathrm{~g} \mathrm{~m}^{-2}\right)$ than other cultivars. The correlation study revealed that the wind speed had significant positive correlation at emergence phase $\left(\mathrm{P}_{1}\right)$ and 3 leaf stage $\left(\mathrm{P}_{2}\right)$. Tmax had significant positive influence and RH-1, RH-II and RF has significant negative influence at button phase $\left(\mathrm{P}_{3}\right)$.

\section{Introduction}

Oil seed crops occupy an important position next to food grains in Indian economy. The oil not only forms an essential part in human diet but also serves as an important raw material for manufacture of various products like flavour enhancers, lubricants etc. Sunflower (Helianthus annuus L.) is one of the most popular members of the family
Asteraceae and is one of the world's most important sources of vegetable oil and It is a rich source of edible oil (40-52\%) having anticholesterol properties (Joksimovic et al., 2006). The native of the sunflower is reported to be Southern parts of USA and Mexico. Sunflower (Helianthus annuus, L), is an important oil crop worldwide. Moreover, it's hardy and superior to sorghum (Sorghum bicolar L) in drought tolerance (Rachid et al., 
1993). Under dryland conditions, sunflower extracts water from deeper soil profile to enable the crop tolerate prolonged dry periods (Unger et al., 1976; Meinke et al., 1993). Also, crop is well adopted by the farming community because of its desirable attributes such as short duration, photoperiod insensitivity, adaptability to wide range of soil and climatic conditions, drought tolerance, lower seed rate, higher seed multiplication ratio and high quality of edible oil $(45-50 \%)$ (Reddy et al., 2007).

\section{Materials and Methods}

The study was conducted at research farm of Zonal Agricultural Research Station, Solapur, Maharashtra State (India) during in year 2013-2017 in the Kharif season. The area is positioned at $75^{\circ} 65^{\prime} \mathrm{N}$ latitude $75^{\circ} 90^{\prime} \mathrm{E}$ longitude and at the altitude of 483.6 meters above the sea level.

The experiment was conducted in split plot design with three replications. Nine treatment combinations were formed considering different cultivars viz., $\mathrm{V}_{1}$ Bhanu, $\mathrm{V}_{2} \mathrm{MSFH}-$ 1 and $\mathrm{V}_{3}$ Phule Bhaskar and sowing windows viz., $\left(\mathrm{S}_{1}\right)$ 2nd fortnight of June $\left(25^{\text {th }}-\mathrm{June}\right)$, $\left(S_{2}\right)$ - 2nd fortnight of July (27 ${ }^{\text {th }}$-July) $S_{3}$-2nd fortnight of August (24 $4^{\text {th }}$-August). The soil comes under the vertisol (medium black) clayey loam in texture and slightly alkaline (pH-7.4) in nature and having the depth up to $90 \mathrm{~cm}$.

The monsoon lasts from June to the end of September, with moderate rainfall. It has an average rainfall of about of $545 \mathrm{~mm}$ per year. The annual maximum and minimum temperature ranged between 25.0 to $43.2^{\circ} \mathrm{C}$ and 7.3 to $27.1^{\circ} \mathrm{C}$, respectively. The gross and net plot sizes were $6.0 \times 4.5 \mathrm{~m}^{2}$ and $4.2 \mathrm{x}$ $3.6 \mathrm{~m}^{2}$, respectively. All the cultivars are were dibbled as per different sowing windows at a spacing of $45 \mathrm{~cm}$ x $20 \mathrm{~cm}$.

\section{Results and Discussion}

\section{Agronomical studies}

The crop sown in second fortnight of July (S2) produced significantly highest grain yield $\left(1708.2 \mathrm{~kg} \mathrm{ha}^{-1}\right)$ and total monetary returns $\left(56320 \mathrm{~kg} \mathrm{ha}^{-1}\right)$ than other dates of sowing. These findings are in confirmative with those reported by Keshta et al., (2006), Dhanasekar et al., (2012) and Khan et al., (2016). In general pearl millet can be sown up to second fortnight of August $\left(\mathrm{S}_{3}\right)$. It was revealed from that the crop sown at second fortnight of July $\left(\mathrm{S}_{2}\right)$ produced significantly higher grain yield and total monetary returns with high degree of sustainability. These results are in close agreement to the findings of Kawade et al., (2018). It might be due to crop sown at second fortnight of July $\left(\mathrm{S}_{2}\right)$ gets sufficient period for its biological and reproductive development and ultimately resulted into higher grain yield and total monetary returns. It also indicates that crop sown at second fortnight of June $\left(\mathrm{S}_{1}\right)$ get sufficient uniform availability of moisture during its life span helps for better yield and monetary benefits. However among the genotypes Phule Bhaskar produced significantly higher grain yield (1446.8 $\mathrm{kg} \mathrm{ha}^{-1}$ ) and total monetary returns (Rs. 47590 $\mathrm{ha}^{-1}$ ). This indicates sustainability of Phule Bhaskar variety over other varieties. This might be due to short duration life span of Phule Bhaskar than other varieties and at reproductive stage $\mathrm{MSFH}-17$ and Bhanu might be faced moisture stress condition (Table 1 and 2).

\section{Meteorological studies}

The mean consumptive use of moisture (CUM) (320 mm) and mean moisture use efficiency (MUE) (5.3 Kg ha $\left.{ }^{-1} \mathrm{~mm}\right)$ was significantly higher recorded by crop sown in second fortnight of July $\left(\mathrm{S}_{2}\right)$ over rest of the 
sowing windows (Table 3). It indicates that early and delay in sowing of crops results in recording low value of CUM and MUE. This might be due to July sown crop gets sufficient period to utilize available soil moisture along with good weather for grain production. Being thermo-sensitive and short day plant, sowing time affect phenology of the crop from adoption to the time of maturity (Kumar and Badiyala, 2005). Phule Bhaskar recorded highest mean value of CUM $(293.1 \mathrm{~mm})$ and MUE (4.8 $\mathrm{Kg} \mathrm{ha}^{-1} \mathrm{~mm}$ ). This indicates that the Phule Bhaskar variety utilized the moisture most efficiently for productions of grains. The mean number of days required to attain the physiological maturity stages recorded higher in July sown crop $\left(\mathrm{S}_{2}\right)$ (Table 4). This might be due to more favorable conditions prevailed in case early sown crop and vice versa. Phule Bhaskar required more mean number of days to attain physiological stages (92 days) than MSFH-17 (84 days) and Bhanu (78 days). This indicates Phule Bhaskar variety required more number of days to attain physiological maturity than other varieties during kharif season under dryland conditions.

The growing degree days (GDD), the function of maximum, minimum and base temperature were presented in Table 5. The crop sown in second fortnight of July (S2) recorded highest mean value of growing degree days (GDD) ( $1042^{0}$ days) than other windows of sowing. It indicates that as a GDD is a function of temperature, during July (S2) sown crop might be grown under high temperature condition and hence recorded highest values of GDD. Further, it is seen that $S_{2}$ sown crop required more growing degree days to attain physiological maturity. Sattar et al., 2017 revealed that variation in phenophase duration caused by changes of sowing dates, which led to early or delayed fulfillment of thermal requirements to attain a particular phenological stage in soybean crop. However, among the varieties the values of mean GDD were higher in Phule Bhaskar $\left(846^{\circ}\right.$ days) followed by MSFH-17 ( $868^{\circ}$ days) and Bhanu $\left(801^{0}\right.$ days) variety. This is due to more duration required by $S_{2}$ sown crop and Phule Bhaskar variety. Further, it was also noticed that the early sown crop not received fairly good amount of rainfall during its growth period due to which soil moisture available was less, however, late sown crop favours due to moisture availability during flowering and grain filling stage which resulted in more duration required for maturity and good yield. In short the second fortnight of July $\left(\mathrm{S}_{2}\right)$ sown crop required more number of days to attain various growth stages. This is due to existence of favourable condition for crop growth and development. This is because the GDD which is function of temperature which in turn is a function of bright sunshine hours.

The mean maximum values of total dry matter were recorded by $S_{2}$ sown crop i.e. $400.8 \mathrm{~g} \mathrm{~m}^{-}$ 2 over rest of the sowing windows. Data revealed that as the delay in sowing of kharif sunflower there is considerable reduction in mean total dry matter. Among the genotype Phule Bhaskar variety recorded highest values of mean total dry matter $\left(404.1 \mathrm{~g} \mathrm{~m}^{-2}\right)$ in almost all the growth stage than the other varieties (Table 6). This indicates that the Phule Bhaskar utilized more efficiently moisture, light and temperature and produced maximum total dry matter by maximum solar radiation interception.

The highest mean values of LAI and RUE recorded by the crop sown in second fortnight of July (1.31 \& $1.63 \mathrm{~g} \mathrm{MJ}^{-1}$ ) (Table $7 \&$ 8) at 50 per cent flowering stage in almost all the sowing dates and genotypes. It was also revealed that with delayed sowing recorded low mean values of LAI and RUE. This indicated that the rate of conversion of light i.e. photosynthetically active radiation (PAR) was considerably high at 50 per cent 
flowering stage, thereafter the conversion rate was declined due to ageing of leaves. Among the sowing windows maximum mean RUE values were higher in July sown crop than late sown crop. Further, it was seen that Phule Bhaskar showed higher values of RUE than MSFH-17 and Bhanu variety for conversion of light into dry matter in all the dates of sowing (Table $7 \& 8$ ). In short the data in respect of mean total dry matter showed that the maximum values were recorded by Phule bhaskar variety in all the windows of sowing. The July $\left(\mathrm{S}_{2}\right)$ sown crop has taken maximum number of days than late sown crops to attain the different growth stages during the crop growth period. This is due to better amount of moisture available and low values of temperature during the crop growth period of July sown crops. The same trend was obtained in case of GDD this indicates that GDD is a function of bright sunshine hours which reflected into a better grain yield..

Table.1 Mean grain yield $\left(\mathrm{kg} \mathrm{ha}^{-1}\right)$ of Kharif Sunflower as influenced by various sowing dates and varieties (2013 to 2017)

\begin{tabular}{|c|c|c|c|c|c|c|c|c|}
\hline Treatment & 2013 & 2014 & 2015 & 2016 & 2017 & Mean & Sur/def $(\%)$ & SYI \\
\hline \multicolumn{9}{|l|}{ Main=3 Sowing dates } \\
\hline $\begin{array}{l}S_{1}=\text { MW } 26 \text { (June 26-July 01) } \\
2^{\text {nd }} \text { fortnight of June }\end{array}$ & 1871.3 & 2109.4 & 491.1 & 1467.4 & 880.4 & 1363.9 & $\begin{array}{l}7.3 \% \text { high over } \\
\text { mean }\end{array}$ & 0.33 \\
\hline $\begin{array}{l}S_{2}=\text { MW } 30 \text { (July 23-29 July) } \\
2^{\text {nd }} \text { fortnight of July }\end{array}$ & 2154.5 & 2392.6 & 827.7 & 1978.9 & 1187.3 & 1708.2 & $\begin{array}{l}34.4 \% \text { high } \\
\text { over mean }\end{array}$ & 0.43 \\
\hline $\begin{array}{l}S_{3}=\text { MW } 35 \text { (August 27-Sept 2) } \\
2^{\text {nd }} \text { fortnight of August }\end{array}$ & 784.2 & 1022.3 & 543.7 & 849.1 & 509.5 & 741.7 & $\begin{array}{l}41.7 \% \text { less over } \\
\text { mean }\end{array}$ & 0.52 \\
\hline Mean & 1603.3 & 1841.4 & 620.8 & 1431.8 & 859.1 & 1271.3 & & 0.41 \\
\hline \multicolumn{9}{|l|}{ Sub $=3$ Varieties } \\
\hline$V_{1}=$ Bhanu & 1322.0 & 1560.1 & 605.9 & 1159.9 & 696.0 & 1068.8 & $\begin{array}{l}15.9 \% \text { less over } \\
\text { mean }\end{array}$ & 0.42 \\
\hline $\mathrm{V}_{2}=\mathrm{MSFH}-17$ & 1590.7 & 1828.8 & 576.8 & 1559.3 & 935.6 & 1298.2 & $\begin{array}{l}2.1 \% \text { high over } \\
\text { mean }\end{array}$ & 0.42 \\
\hline V3= Phule Bhaskar & 1897.3 & 2135.3 & 679.7 & 1576.1 & 945.7 & 1446.8 & $\begin{array}{l}13.8 \% \text { high } \\
\text { over mean }\end{array}$ & 0.39 \\
\hline Mean & 1603.3 & 1841.4 & 620.8 & 1431.8 & 859.1 & 1271.3 & & 0.41 \\
\hline \multicolumn{9}{|l|}{ Sub-Sub $=2$ Treatments } \\
\hline T1=Protected & 1789.0 & 2027.1 & 718.3 & 1600.6 & 960.3 & 1419.0 & $\begin{array}{l}11.6 \% \text { high } \\
\text { over mean }\end{array}$ & 0.43 \\
\hline T2=Unprotected & 1417.7 & 1655.8 & 523.3 & 1263.0 & 757.8 & 1123.5 & $\begin{array}{l}11.6 \% \text { less over } \\
\text { mean }\end{array}$ & 0.39 \\
\hline Mean & 1603.3 & 1841.4 & 620.8 & 1431.8 & 859.1 & 1271.3 & & 0.41 \\
\hline S.E.. \pm (Sowing dates) & 45.37 & 45.4 & 14.8 & 36.8 & 22.1 & 125.5 & & \\
\hline C.D. at $5 \%$ & 178.16 & 178.2 & 58.1 & 144.5 & 86.7 & 409.2 & & \\
\hline S.E. \pm (Varieties) & 78.80 & 78.8 & 26.4 & 35.6 & 21.4 & 41.5 & & \\
\hline C.D. at $5 \%$ & 242.80 & 242.8 & 81.3 & 109.6 & 65.8 & 121.1 & & \\
\hline S.E. $\pm($ SD X V) & 136.48 & 136.5 & 45.7 & 61.6 & 37.0 & 71.8 & & \\
\hline C.D. at $5 \%$ & NS & NS & NS & 189.9 & 113.9 & NS & & \\
\hline S.E. \pm (Treatment) & 4.51 & 4.5 & 18.4 & 45.6 & 27.4 & 18.4 & & \\
\hline C.D. at $5 \%$ & 13.41 & 13.4 & 54.7 & 135.6 & 81.3 & 52.7 & & \\
\hline S.E. \pm (SD x Treatment) & 13.54 & 13.5 & 31.9 & 79.0 & 47.4 & 31.8 & & \\
\hline C.D. at $5 \%$ & 40.22 & 40.2 & 94.8 & NS & NS & 91.2 & & \\
\hline S.E. \pm (Treatment X Variety) & 13.54 & 13.5 & 31.9 & 79.0 & 47.4 & 31.8 & & \\
\hline C.D. at $5 \%$ & NS & NS & NS & NS & NS & NS & & \\
\hline
\end{tabular}


Table.2 Mean total monetary returns of Kharif Sunflower as influenced by various sowing dates and varieties (2013 to 2017)

\begin{tabular}{|c|c|c|c|c|c|c|c|}
\hline Treatment & 2013 & 2014 & 2015 & 2016 & 2017 & Mean & SYI \\
\hline \multicolumn{8}{|l|}{ Main=3 Sowing dates } \\
\hline $\begin{array}{l}S_{1}=\text { MW } 26 \text { (June 26-July01) } \\
2^{\text {nd }} \text { fortnight of June }\end{array}$ & 58730 & 55326 & 21925 & 55762 & 33457 & 45040 & 0.49 \\
\hline $\begin{array}{l}S_{2}=\text { MW } 30 \text { (July. 23-29) } 2^{\text {nd }} \\
\text { fortnight of July }\end{array}$ & 66444 & 61624 & 33216 & 75197 & 45118 & 56320 & 0.52 \\
\hline $\begin{array}{l}S_{3}=\text { MW } 35 \text { (August 27-Sept 2) } \\
2^{\text {nd }} \text { fortnight of August }\end{array}$ & 26005 & 28037 & 20650 & 32267 & 19360 & 25264 & 0.62 \\
\hline Mean & 50393 & 48329 & 25263 & 54409 & 32645 & 42208 & 0.54 \\
\hline \multicolumn{8}{|l|}{ Sub $=3$ Varieties } \\
\hline$V_{1}=$ Bhanu & 42303 & 41646 & 25061 & 44077 & 26446 & 35907 & 0.60 \\
\hline$V_{2}=$ MSFH-17 & 49736 & 47736 & 23357 & 59255 & 35553 & 43128 & 0.49 \\
\hline V3= Phule Bhaskar & 59140 & 55606 & 27372 & 59894 & 35936 & 47590 & 0.55 \\
\hline Mean & 50393 & 48329 & 25263 & 54409 & 32645 & 42208 & 0.54 \\
\hline \multicolumn{8}{|l|}{ Sub-Sub $=2$ Treatments } \\
\hline T1=Protected & 56132 & 53140 & 29039 & 60822 & 36493 & 47125 & 0.55 \\
\hline T2=Unprotected & 44654 & 43518 & 21487 & 47995 & 28797 & 37290 & 0.54 \\
\hline Mean & 50393 & 48329 & 25263 & 54409 & 32645 & 42208 & 0.54 \\
\hline S.E. \pm (Sowing dates) & 1360.63 & 1133.8 & 559.3 & 1398.4 & 839.0 & 3319.3 & \\
\hline C.D. at $5 \%$ & 5342.49 & 4451.7 & 2195.9 & 5490.8 & 3294.5 & 10824.7 & \\
\hline S.E. \pm (Varieties) & 2351.68 & 1957.7 & 1009.5 & 1352.2 & 811.3 & 1291.5 & \\
\hline C.D. at $5 \%$ & 7246.23 & 6032.4 & 3110.5 & 4166.5 & 2499.9 & 3769.5 & \\
\hline S.E. $\pm($ SD X V) & 4073.22 & 3390.9 & 1748.5 & 2342.1 & 1405.2 & 2236.9 & \\
\hline C.D. at $5 \%$ & NS & NS & NS & 7216.7 & 4330.0 & NS & \\
\hline S.E. \pm (Treatment) & 134.59 & 112.0 & 681.6 & 1733.7 & 1040.2 & 530.1 & \\
\hline C.D. at $5 \%$ & 399.87 & 332.9 & 2025.2 & 5151.1 & 3090.7 & 1520.3 & \\
\hline S.E. \pm (SD x Treatment) & 403.76 & 336.1 & 1180.6 & 3002.9 & 1801.7 & 918.1 & \\
\hline C.D. at $5 \%$ & 1199.62 & 998.6 & 3507.7 & NS & NS & 2633.2 & \\
\hline S.E. \pm (Treatment X Variety) & 403.76 & 336.1 & 1180.6 & 3002.9 & 1801.7 & 918.1 & \\
\hline C.D. at $5 \%$ & NS & NS & NS & NS & NS & NS & \\
\hline
\end{tabular}

Table.3 CUM and MUE as influenced by different treatments in Kharif sunflower (2013 to 2017)

\begin{tabular}{|c|c|c|c|c|c|c|c|}
\hline Treatment & $\begin{array}{c}\mathbf{G Y} \\
\left(\mathbf{k g ~ h a}^{-1}\right)\end{array}$ & $\begin{array}{c}\mathbf{C U M} \\
(\mathbf{m m})\end{array}$ & $\begin{array}{c}\mathbf{M U E} \\
\left(\mathbf{k g ~ h a}^{-1} \mathbf{m m}\right)\end{array}$ & Treatment & $\begin{array}{c}\mathbf{G Y} \\
\left(\mathbf{k g ~ h a} \mathbf{- 1}^{-1}\right)\end{array}$ & $\begin{array}{c}\mathbf{C U M} \\
(\mathbf{m m})\end{array}$ & $\begin{array}{c}\mathbf{M U E} \\
\left(\mathbf{k g} \mathbf{h a}^{-1} \mathbf{m m}\right)\end{array}$ \\
\hline $\mathrm{S}_{1} \mathrm{~V}_{1} \mathrm{~T}_{1}$ & 1305 & 268 & 4.9 & $\mathrm{~S}_{2} \mathrm{~V}_{2} \mathrm{~T}_{2}$ & 1716 & 317 & 5.4 \\
\hline $\mathrm{S}_{1} \mathrm{~V}_{1} \mathrm{~T}_{2}$ & 1013 & 242 & 4.2 & $\mathrm{~S}_{2} \mathrm{~V}_{3} \mathrm{~T}_{1}$ & 1977 & 337 & 5.9 \\
\hline $\mathrm{S}_{1} \mathrm{~V}_{2} \mathrm{~T}_{1}$ & 1495 & 308 & 4.9 & $\mathrm{~S}_{2} \mathrm{~V}_{3} \mathrm{~T}_{2}$ & 1822 & 316 & 5.8 \\
\hline $\mathrm{S}_{1} \mathrm{~V}_{2} \mathrm{~T}_{2}$ & 1298 & 298 & 4.4 & $\mathrm{~S}_{3} \mathrm{~V}_{1} \mathrm{~T}_{1}$ & 782 & 254 & 3.1 \\
\hline $\mathrm{S}_{1} \mathrm{~V}_{3} \mathrm{~T}_{1}$ & 1700 & 312 & 5.4 & $\mathrm{~S}_{3} \mathrm{~V}_{1} \mathrm{~T}_{2}$ & 424 & 215 & 2.0 \\
\hline $\mathrm{S}_{1} \mathrm{~V}_{3} \mathrm{~T}_{2}$ & 1373 & 280 & 4.9 & $\mathrm{~S}_{3} \mathrm{~V}_{2} \mathrm{~T}_{1}$ & 941 & 281 & 3.3 \\
\hline $\mathrm{S}_{2} \mathrm{~V}_{1} \mathrm{~T}_{1}$ & 1604 & 326 & 4.9 & $\mathrm{~S}_{3} \mathrm{~V}_{2} \mathrm{~T}_{2}$ & 494 & 192 & 2.6 \\
\hline $\mathrm{S}_{2} \mathrm{~V}_{1} \mathrm{~T}_{2}$ & 1285 & 291 & 4.4 & $\mathrm{~S}_{3} \mathrm{~V}_{3} \mathrm{~T}_{1}$ & 1123 & 293 & 3.8 \\
\hline $\mathrm{S}_{2} \mathrm{~V}_{2} \mathrm{~T}_{1}$ & 1846 & 333 & 5.5 & $\mathrm{~S}_{3} \mathrm{~V}_{3} \mathrm{~T}_{2}$ & 686 & 221 & 3.1 \\
\hline
\end{tabular}


Table.4 Number days required to attain phenological stages as influenced by sowing dates in sunflower (2013 to 2017)

\begin{tabular}{|l|c|c|c|c|c|c|c|}
\hline $\begin{array}{l}\text { Sowing } \\
\text { Time }\end{array}$ & Emer. & $\mathbf{4}$ leaf & Button & $\begin{array}{r}\mathbf{5 0} \% \\
\text { flowering }\end{array}$ & $\begin{array}{c}\text { Soft } \\
\text { dough }\end{array}$ & $\begin{array}{c}\text { Hard } \\
\text { dough }\end{array}$ & $\begin{array}{c}\text { Phy. } \\
\text { Maturity }\end{array}$ \\
\hline $\mathbf{S}_{\mathbf{1}} \mathbf{V}_{\mathbf{1}}$ & 8 & 6 & 25 & 11 & 8 & 8 & 7 \\
\hline Cumulative & 8 & 14 & 39 & 50 & 58 & 66 & 73 \\
\hline $\mathbf{S}_{\mathbf{1}} \mathbf{V}_{\mathbf{2}}$ & 7 & 8 & 26 & 10 & 8 & 7 & 6 \\
\hline Cumulative & 7 & 15 & 41 & 51 & 59 & 66 & 72 \\
\hline $\mathbf{S}_{\mathbf{1}} \mathbf{V}_{\mathbf{3}}$ & 6 & 7 & 24 & 11 & 7 & 6 & 6 \\
\hline Cumulative & 6 & 13 & 37 & 48 & 55 & 61 & 67 \\
\hline $\mathbf{S}_{\mathbf{2}} \mathbf{V}_{\mathbf{1}}$ & 7 & 7 & 28 & 12 & 8 & 7 & 7 \\
\hline Cumulative & 7 & 14 & 42 & 54 & 62 & 69 & 76 \\
\hline $\mathbf{S}_{\mathbf{2}} \mathbf{V}_{\mathbf{2}}$ & 8 & 7 & 30 & 13 & 8 & 10 & 8 \\
\hline Cumulative & 8 & 15 & 45 & 58 & 66 & 76 & 84 \\
\hline $\mathbf{S}_{\mathbf{2}} \mathbf{V}_{\mathbf{3}}$ & 8 & 8 & 32 & 13 & 11 & 12 & 8 \\
\hline Cumulative & 8 & 16 & 48 & 61 & 72 & 84 & 92 \\
\hline $\mathbf{S}_{\mathbf{3}} \mathbf{V}_{\mathbf{1}}$ & 7 & 6 & 27 & 11 & 7 & 10 & 7 \\
\hline Cumulative & 7 & 13 & 40 & 51 & 58 & 68 & 75 \\
\hline $\mathbf{S}_{\mathbf{3}} \mathbf{V}_{\mathbf{2}}$ & 6 & 7 & 29 & 12 & 8 & 8 & 8 \\
\hline Cumulative & 6 & 13 & 42 & 54 & 62 & 70 & 78 \\
\hline $\mathbf{S}_{\mathbf{3}} \mathbf{V}_{\mathbf{3}}$ & 7 & 10 & 30 & 11 & 9 & 8 & 7 \\
\hline Cumulative & 7 & 17 & 47 & 58 & 67 & 75 & 82 \\
\hline
\end{tabular}

Table.5 Growing degree days required to attain phenological stages as influenced by sowing dates in sunflower (2013 to 2017)

\begin{tabular}{|c|c|c|c|c|c|c|c|}
\hline $\begin{array}{c}\text { Sowing } \\
\text { Time }\end{array}$ & Emer. & 4 leaf & Button & $\begin{array}{c}\mathbf{5 0} \text { \% } \\
\text { flowering }\end{array}$ & $\begin{array}{c}\text { Soft } \\
\text { dough }\end{array}$ & $\begin{array}{c}\text { Hard } \\
\text { dough }\end{array}$ & $\begin{array}{c}\text { Phy. } \\
\text { Maturity }\end{array}$ \\
\hline $\mathbf{S}_{\mathbf{1}} \mathbf{V}_{\mathbf{1}}$ & 83 & 75 & 185 & 54 & 68 & 101 & 84 \\
\hline Cumulative & 83 & 158 & 343 & 397 & 465 & 566 & 650 \\
\hline $\mathbf{S}_{\mathbf{1}} \mathbf{V}_{\mathbf{2}}$ & 86 & 93 & 240 & 124 & 86 & 176 & 90 \\
\hline Cumulative & 86 & 179 & 419 & 543 & 629 & 805 & 895 \\
\hline $\mathbf{S}_{\mathbf{1}} \mathbf{V}_{\mathbf{3}}$ & 64 & 90 & 72 & 55 & 87 & 193 & 103 \\
\hline Cumulative & 64 & 154 & 226 & 281 & 368 & 561 & 664 \\
\hline $\mathbf{S}_{\mathbf{2}} \mathbf{V}_{\mathbf{1}}$ & 84 & 82 & 192 & 134 & 185 & 210 & 116 \\
\hline Cumulative & 84 & 166 & 358 & 492 & 677 & 887 & 1003 \\
\hline $\mathbf{S}_{\mathbf{2}} \mathbf{V}_{\mathbf{2}}$ & 84 & 101 & 286 & 111 & 106 & 186 & 125 \\
\hline Cumulative & 84 & 185 & 471 & 582 & 688 & 874 & 999 \\
\hline $\mathbf{S}_{\mathbf{2}} \mathbf{V}_{\mathbf{3}}$ & 83 & 126 & 289 & 145 & 165 & 210 & 107 \\
\hline Cumulative & 83 & 209 & 498 & 643 & 808 & 1018 & 1125 \\
\hline $\mathbf{S}_{\mathbf{3}} \mathbf{V}_{\mathbf{1}}$ & 88 & 107 & 186 & 70 & 85 & 105 & 109 \\
\hline Cumulative & 88 & 195 & 381 & 451 & 536 & 641 & 750 \\
\hline $\mathbf{S}_{\mathbf{3}} \mathbf{V}_{\mathbf{2}}$ & 72 & 91 & 186 & 88 & 86 & 105 & 84 \\
\hline Cumulative & 72 & 163 & 349 & 437 & 523 & 628 & 712 \\
\hline $\mathbf{S}_{\mathbf{3}} \mathbf{V}_{\mathbf{3}}$ & 86 & 106 & 230 & 59 & 82 & 102 & 85 \\
\hline Cumulative & 86 & 192 & 422 & 481 & 563 & 665 & 750 \\
\hline
\end{tabular}


Table.6 Periodical dry matter $\left(\mathrm{g} \mathrm{m}^{-2}\right)$ and its partitioning into different parts of sunflower (2013 to 2017)

\begin{tabular}{|c|c|c|c|c|c|c|}
\hline Sowing & \multicolumn{7}{|c|}{ Growth Stage } \\
\hline Date & 4 Leaf & Button & $\mathbf{5 0 \%}$ flow. & Soft Dough & Hard Dough & Phy. Maturity \\
\hline $\mathrm{S}_{1} \mathrm{~V}_{1}$ & 1.8 & 62.3 & 111.0 & 166.4 & 217.2 & 285.2 \\
\hline $\mathrm{S}_{1} \mathrm{~V}_{2}$ & 1.6 & 73.3 & 116.2 & 186.0 & 229.0 & 318.9 \\
\hline $\mathrm{S}_{1} \mathrm{~V}_{3}$ & 1.9 & 83.8 & 134.1 & 211.0 & 276.5 & 357.2 \\
\hline $\mathrm{S}_{2} \mathrm{~V}_{1}$ & 1.9 & 88.5 & 149.1 & 235.7 & 315.6 & 369.4 \\
\hline $\mathrm{S}_{2} \mathrm{~V}_{2}$ & 2.3 & 93.5 & 156.3 & 260.8 & 334.9 & 398.6 \\
\hline $\mathrm{S}_{2} \mathrm{~V}_{3}$ & 2.0 & 106.7 & 174.6 & 295.0 & 373.3 & 434.4 \\
\hline $\mathrm{S}_{3} \mathrm{~V}_{1}$ & 1.6 & 73.5 & 142.3 & 223.9 & 317.7 & 356.4 \\
\hline $\mathrm{S}_{3} \mathrm{~V}_{2}$ & 1.7 & 91.8 & 150.3 & 254.9 & 337.7 & 387.5 \\
\hline $\mathrm{S}_{3} \mathrm{~V}_{3}$ & 2.0 & 99.8 & 168.4 & 279.3 & 364.5 & 420.8 \\
\hline
\end{tabular}

Table7 Leaf area index as influenced by sowing dates in sunflower (2013 to 2017)

\begin{tabular}{|l|c|c|c|c|c|c|}
\hline Sowing & \multicolumn{7}{|c|}{ Growth Stage } \\
\hline Date & 4 Leaf & Button & $\mathbf{5 0 \%}$ flow. & Soft Dough & Hard Dough & Phy. Maturity \\
\hline $\mathrm{S}_{1} \mathrm{~V}_{1}$ & 0.019 & 0.33 & 1.06 & 0.48 & 0.26 & 0.03 \\
\hline $\mathrm{S}_{1} \mathrm{~V}_{2}$ & 0.020 & 0.32 & 0.90 & 0.55 & 0.25 & 0.06 \\
\hline $\mathrm{S}_{1} \mathrm{~V}_{3}$ & 0.018 & 0.43 & 1.03 & 0.68 & 0.43 & 0.06 \\
\hline $\mathrm{S}_{2} \mathrm{~V}_{1}$ & 0.020 & 0.52 & 1.27 & 0.55 & 0.53 & 0.15 \\
\hline $\mathrm{S}_{2} \mathrm{~V}_{2}$ & 0.021 & 0.48 & 1.23 & 0.57 & 0.47 & 0.11 \\
\hline $\mathrm{S}_{2} \mathrm{~V}_{3}$ & 0.023 & 0.59 & 1.43 & 0.78 & 0.61 & 0.21 \\
\hline $\mathrm{S}_{3} \mathrm{~V}_{1}$ & 0.019 & 0.35 & 0.96 & 0.43 & 0.33 & 0.03 \\
\hline $\mathrm{S}_{3} \mathrm{~V}_{2}$ & 0.018 & 0.47 & 1.10 & 0.59 & 0.43 & 0.11 \\
\hline $\mathrm{S}_{3} \mathrm{~V}_{3}$ & 0.017 & 0.44 & 1.00 & 0.64 & 0.44 & 0.06 \\
\hline
\end{tabular}

Table.8 Radiation use efficiency ( $\mathrm{g} \mathrm{MJ}^{-1}$ ) by sowing dates in sunflower (2013 to 2017)

\begin{tabular}{|l|c|c|c|c|c|c|}
\hline Sowing & \multicolumn{6}{|c|}{ Growth Stage } \\
\hline Date & 4 Leaf & Button & $\mathbf{5 0 \%}$ flow. & Soft Dough & Hard Dough & Phy. Maturity \\
\hline $\mathrm{S}_{1} \mathrm{~V}_{1}$ & 0.09 & 0.46 & 1.16 & 0.79 & 0.84 & 0.3 \\
\hline $\mathrm{S}_{1} \mathrm{~V}_{2}$ & 0.09 & 0.49 & 1.15 & 0.81 & 0.76 & 0.4 \\
\hline $\mathrm{S}_{1} \mathrm{~V}_{3}$ & 0.11 & 0.55 & 1.55 & 0.86 & 0.72 & 0.46 \\
\hline $\mathrm{S}_{2} \mathrm{~V}_{1}$ & 0.12 & 0.53 & 1.53 & 1.27 & 1.25 & 0.29 \\
\hline $\mathrm{S}_{2} \mathrm{~V}_{2}$ & 0.12 & 0.57 & 1.64 & 1.32 & 1.29 & 0.47 \\
\hline $\mathrm{S}_{2} \mathrm{~V}_{3}$ & 0.13 & 0.63 & 1.71 & 1.40 & 1.31 & 0.52 \\
\hline $\mathrm{S}_{3} \mathrm{~V}_{1}$ & 0.09 & 0.42 & 0.93 & 0.66 & 0.63 & 0.39 \\
\hline $\mathrm{S}_{3} \mathrm{~V}_{2}$ & 0.09 & 0.45 & 0.93 & 0.69 & 0.62 & 0.42 \\
\hline $\mathrm{S}_{3} \mathrm{~V}_{3}$ & 0.10 & 0.49 & 1.46 & 0.73 & 0.71 & 0.46 \\
\hline
\end{tabular}


Table.9 Correlation coefficient between grain yield and different weather parameters during different phenophases of kharif sunflower

\begin{tabular}{|l|l|l|l|l|l|l|l|l|}
\hline Phenophase & $\begin{array}{l}\text { Tmax } \\
(\mathbf{0} \mathbf{C})\end{array}$ & $\begin{array}{l}\text { Tmin } \\
\left.\mathbf{(}^{\mathbf{0}} \mathbf{C}\right)\end{array}$ & $\begin{array}{l}\mathbf{R H - 1} \\
\mathbf{( \% )}\end{array}$ & $\begin{array}{l}\mathbf{R H - 2} \\
\mathbf{( \% )}\end{array}$ & $\begin{array}{l}\text { WS } \\
(\mathbf{k m p h})\end{array}$ & RF (mm) & $\begin{array}{l}\text { SS } \\
\left(\mathbf{h r s ~ d a y} \mathbf{~}^{-1}\right)\end{array}$ & $\begin{array}{l}\text { EVP } \\
(\mathbf{m m})\end{array}$ \\
\hline P1 & -0.260 & 0.184 & -0.221 & 0.342 & $0.694^{* *}$ & -0.071 & -0.464 & 0.331 \\
\hline P2 & -0.233 & 0.207 & -0.428 & -0.157 & $0.512^{*}$ & -0.044 & -0.326 & 0.216 \\
\hline P3 & $0.626^{* *}$ & 0.334 & $-0.732^{* *}$ & $-0.560^{*}$ & 0.400 & $-0.704^{* *}$ & -0.020 & 0.428 \\
\hline P4 & -0.453 & $0.508^{*}$ & $0.544^{*}$ & $0.623^{* *}$ & 0.336 & 0.248 & $-0.519^{*}$ & $0.544^{*}$ \\
\hline P5 & $-0.509^{*}$ & $0.592^{* *}$ & $0.710^{* *}$ & $0.657^{* *}$ & 0.154 & $0.754^{* *}$ & -0.450 & $0.576^{*}$ \\
\hline P6 & 0.342 & 0.377 & 0.311 & 0.146 & 0.042 & 0.281 & 0.074 & $0.763^{* *}$ \\
\hline P7 & 0.353 & 0.268 & 0.094 & 0.182 & -0.041 & -0.040 & -0.049 & 0.246 \\
\hline
\end{tabular}

Table.10 Stepwise multiple regression of different weather parameters with yield of kharif sunflower at soft dough stage (2012 to 2017)

\begin{tabular}{|l|l|c|l|}
\hline Sr. No. & Weather parameter & Regression coefficient & $\mathbf{R}^{\mathbf{2}}$ \\
\hline $\mathbf{1}$ & Intercept & -87.801 & $\mathbf{0 . 7 0}$ \\
\hline $\mathbf{2}$ & Minimum Temperature $\left(\mathrm{T}_{\min }\right)$ & -3.864 & \\
\hline $\mathbf{3}$ & Relative Humidity $(\mathrm{RH}-1)$ & 2.338 & \\
$\mathbf{4}$ & Relative Humidity $(\mathrm{RH}-2)$ & -0.185 & \\
\hline $\mathbf{5}$ & Rainfall $(\mathrm{RF})$ & -0.077 & \\
\hline $\mathbf{6}$ & Epan & $\mathbf{0 . 3 7 2}$ & \\
\hline
\end{tabular}

Table.11 Observed and predicted yield by using linear regression equations

\begin{tabular}{|c|c|c|c|c|}
\hline Treatment & Actual Yield & $\begin{array}{l}\text { Predicted } \\
\text { Yield }\end{array}$ & Residuals & $\begin{array}{l}\text { Standardized } \\
\text { residual }\end{array}$ \\
\hline \multicolumn{5}{|l|}{ Main treatment - Sowing time } \\
\hline $\begin{array}{l}S_{1}=\text { MW } 26 \text { (June 26-July 01) } \\
2^{\text {nd }} \text { fortnight of June }\end{array}$ & 1481.8 & 1473.0 & 8.8 & -0.248 \\
\hline $\begin{array}{l}S_{2}=\text { MW } 30(\text { July 23-29) } \\
2^{\text {nd }} \text { fortnight of July }\end{array}$ & 1763.5 & 1744.8 & 18.7 & 1.101 \\
\hline $\begin{array}{l}S_{3}=\text { MW } 35 \text { (August 27-Sept 2) } \\
2^{\text {nd }} \text { fortnight of August }\end{array}$ & 995.1 & 990.8 & 4.3 & -0.853 \\
\hline \multicolumn{5}{|l|}{ Sub treatment - variety } \\
\hline$V_{1}=$ Bhanu & 1228.1 & 1298.4 & -70.3 & -1.007 \\
\hline $\mathbf{V}_{2}=$ MSFH-17 & 1425.6 & 1335.2 & 90.4 & 0.993 \\
\hline V3= Phule Bhaskar & 1586.7 & 1575.0 & 11.7 & 0.014 \\
\hline \multicolumn{5}{|l|}{ Sub-Sub $=2$ Treatments } \\
\hline T1=Protected & 1601.3 & 1402.9 & 198.4 & 0.707 \\
\hline T2=Unprotected & 1225.7 & 1402.9 & -177.2 & -0.707 \\
\hline \multicolumn{5}{|c|}{$\begin{array}{l}\sqrt{\text { Yield }}=-87.801+\left(-3.864 \times \mathrm{T}_{\min }\right)+(2.338 \times \mathrm{RH}-1)+(-0.185 \times \mathrm{RH}-2)+(-0.077 \times \mathrm{RF})+ \\
\text { Standard Residual }>3 \text { and }<-3 \text { is outleter, } \\
\mathrm{T}_{\min }=\text { Min. Temperature }\left({ }^{\circ} \mathrm{C}\right) \\
\mathrm{RH}-1=\text { Morning relative humidity }(\%) \\
\mathrm{RH}-2=\text { Evening relative humidity }(\%) \\
\mathrm{RF} \quad=\text { Rainfall }(\mathrm{mm})\end{array}$} \\
\hline
\end{tabular}



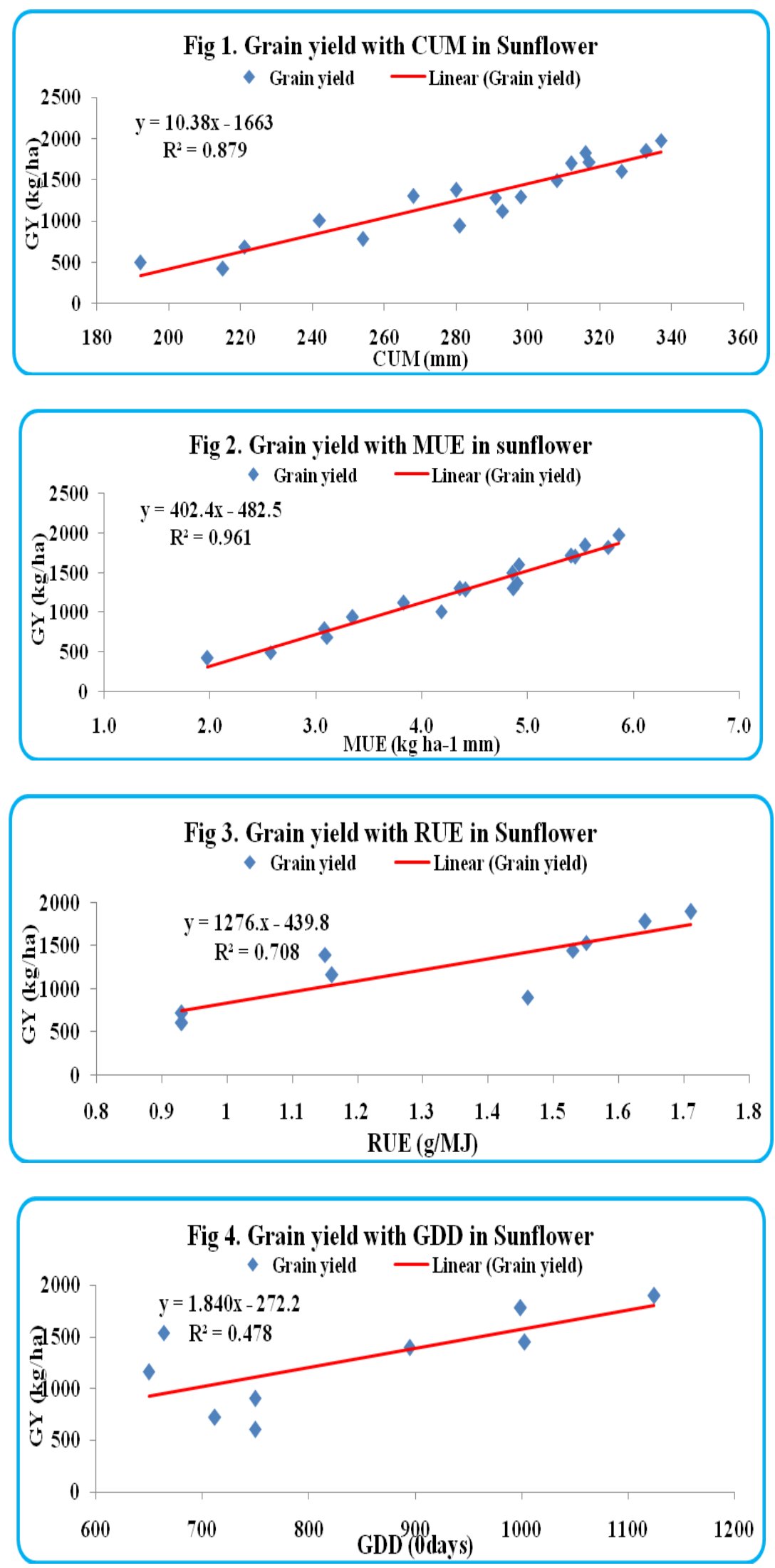

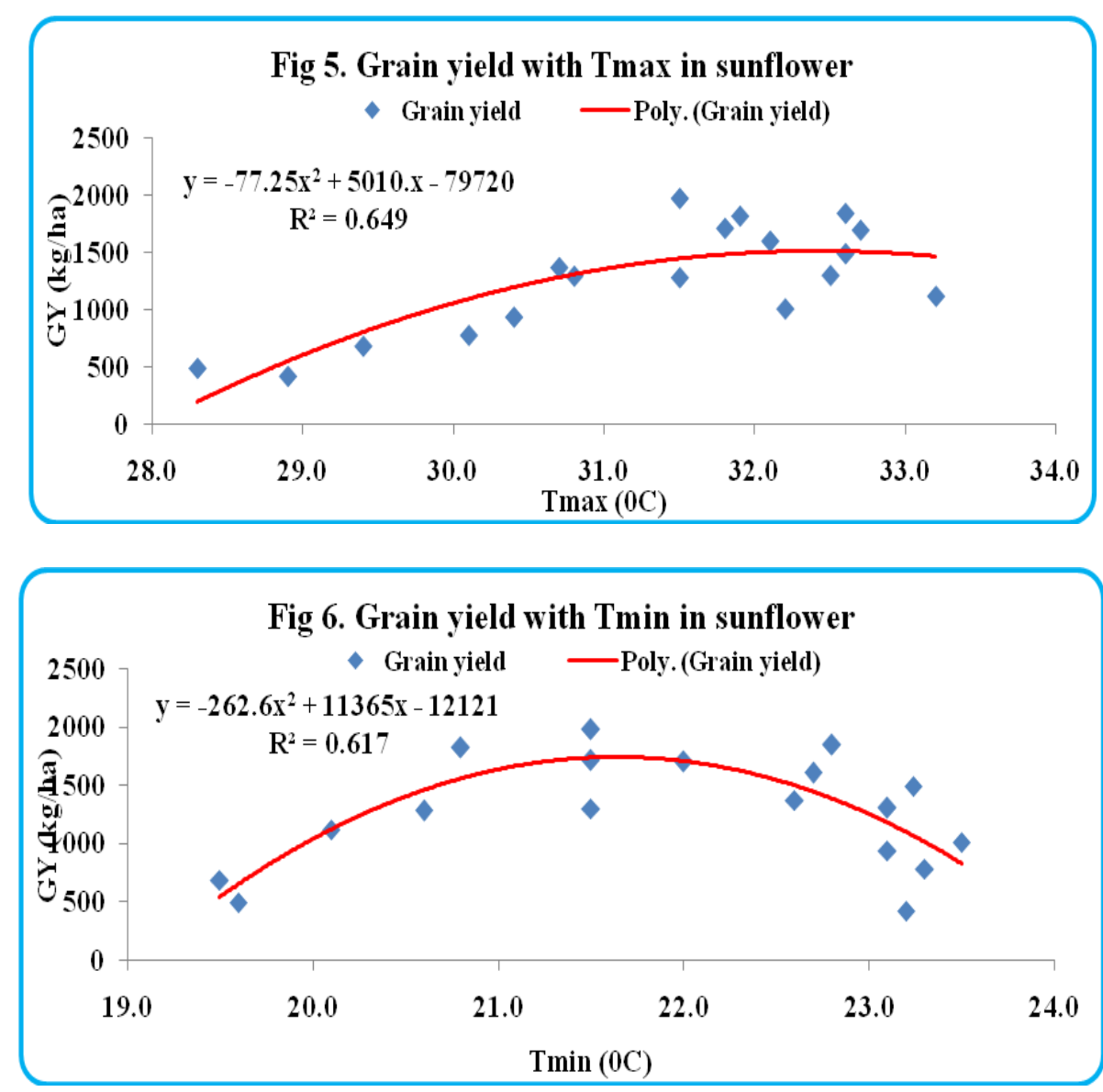

\section{Correlation regression studies}

The weather parameter influence their contribution and performance in kharif sunflower crop sown in different sowing windows were assessed in tenure of phase wise correlation and regression (Table 9-11).

The influences of weather parameter and agrometeorological indices on performance of kharif sunflower crop sown at different windows with different varieties were assured in terms of phase-wise correlation of grain yield with mentioned weather parameters. It is revealed that the wind speed had significant positive correlation at emergence phase $\left(\mathrm{P}_{1}\right)$ and 3 leaf stage $\left(\mathrm{P}_{2}\right)$. Tmax had significant positive influence and RH-1, RH-2 and RF has significant negative influence at button phase $\left(\mathrm{P}_{3}\right)$. Significant positive correlation was found with Tmin, RH-1, RH-2 and Epan while BSS has significant negative correlation at $50 \%$ flowering stage (P4). Tmax had significant negative influence and Tmin, RH1, RH-2, RF and Epan has significant positive influence at soft dough phase (P5) while at hard dough stage $\left(\mathrm{P}_{6}\right)$ Epan had significant positive association with grain yield.

Significant negative association with grain yield by Tmin, RH-1, RH-2 and RF at button phase (P3) indicates that at early growth stages kharif sunflower not favour moisture stress condition. Significant positive association with grain yield at $50 \%$ flowering stage by Tmin, RH-1, RH-2, RF and Epan indicates kharif sunflower responds well to available moisture and low temperature conditions. It is revealed that button phase $\left(\mathrm{P}_{3}\right)$ and $50 \%$ flowering stage $\left(\mathrm{P}_{4}\right)$ are more crucial growth stages to contribute grain production. 
It is observed that the significantly positive correlation (Table 9) of weather parameters namely Tmin, RH-1, RH-2 and RF with grain yield at soft dough phase. The predicted grain yield and actual pooled grain yield is presented in Table 11 . The regression equation is developed by using this weather parameters i.e. $\quad \sqrt{ }$ Yield $=-87.801+\quad($ $3.864 \times \mathrm{Tmin})+(2.338 \times \mathrm{RH}-1)+(-0.185 \times \mathrm{RH}-$ $2)+(-0.077 \times \mathrm{RF})+(0.372 \times$ Epan $)$. This equation is helpful to predict grain yield after completion of soft dough phase (P5).

The consumptive use of moisture (CUM) during total growth period of kharif Sunflower Fig. 1 showed a linear relationship with grain yield $\left(y=10.389 x-1663 R^{2}=0.88\right)$. The CUM of $320 \mathrm{~mm}$ was found to be optimum for getting higher grain yield. The moisture use efficiency (MUE) during total growth period of kharif sunflower Fig. 2 showed a linear relationship with grain yield $\left.\mathrm{y}=402.41 \mathrm{x}-482.52 \mathrm{R}^{2}=0.96\right)$. The MUE of 4.50 to $5.50 \mathrm{~kg}$ ha $\mathrm{mm}^{-1}$ was found to be optimum for getting higher grain yield.

The RUE studies depicted in Fig. 3 showed linear relationship with grain yield. This indicated that radiation interception is directly related with grain yield $(y=1276.9 x-439.81$ $\left.\mathrm{R}^{2}=0.71\right)$. The figure showed that if RUE increases from 1.5 to $1.7 \mathrm{~g} \mathrm{mj}^{-1}$ it increases the yield from 06 to $10 \mathrm{q} \mathrm{ha}^{-1}$. This indicated that every increase of $0.1 \mathrm{gmj}^{-1}$ of energy there is increase of $0.9 \mathrm{q} \mathrm{ha}^{-1}$ of grain yield of sunflower.

The GDD was correlated with the grain yield of sunflower and depicted in Fig. 4. It showed a linear relationship with grain yield $\left(y=1.8405 x-272.26 R^{2}=0.48\right)$. This indicated that with increase of GDD there was increase in grain yield upto 1000 GDD.

The $\mathrm{T}_{\max }$ was correlated with the grain yield of sunflower and depicted in Fig. 5. It showed a polynomial relationship with grain yield (y $=-77.257 \mathrm{x}^{2}+5010.4 \mathrm{x}-79720$ $\left.\mathrm{R}^{2}=0.65\right)$. This indicated that with increase of $\mathrm{T}_{\max }$ there was increase in grain yield upto $31.6^{0} \mathrm{C}$. The $\mathrm{T}_{\min }$ was correlated with the grain yield of sunflower and depicted in Fig. 6. It showed a polynomial relationship with grain yield $\left(y=-262.6 x^{2}+11365 x-121216 R^{2}=0.62\right)$. This indicated that with increase of $\mathrm{T}_{\min }$ there was increase in grain yield upto $21.6^{\circ} \mathrm{C}$

\section{References}

Joksimovic, J., Atlagic Jovanka, Marinkovic, R. and Jovanovi, D., 2006, Genetic control of oleic and linoleic acid contents in sunflower. Helia, 29(44): 33-40.

Reddy, N. Y. A., Shaanker, R .U., Prasad, T. G. and Kumar, M. U. 2003. Physiological approaches to improve harvest index and productivity in sunflower. Helia., 26: 81-90.

Rachid, F., Kirkham, M.B., Stone, L.R. \& Kanemasu, E.T. 1993. Soil water depletion by sunflower and sorghum under rainfed conditions. Agricultural Water Management 24, 49-62.

Meinke, H., Hammer, G.L. and Wart, P. 1993. Potential soil water extraction by sunflower on a range of soils. Field Crops Research 32, 59-81.

Unger, P.W., Allen, R.R., Jones, O.R., Mathers, A.C. and Stewart, B.A. 1976. Sunflower research in the southern High Plains. A Progress Report. Proceeding Sunflower Forum. Fargo, ND. 1,24-29.

Kawade,M.B, Jadhav, D.B., and Arshewar, S.P. 2018. Effect of Micronutrients on Growth, Yield and Quality of Sunflower in Kharif Season. International Journal of Current Microbiology and Applied Sciences., 6: 2189-2196.

Keshta, M.M., Rizk, T.Y. and Abdou, E.T. 2006. Sunflower response to mineral nitrogen, organic and bio-fertilizers 
under two different levels of salinity. Proc. 17th International Sunflower Conference, Córdoba, Spain.

Dhanasekar, R. and Dhandapani, R. 2012. Effect of biofertilizers on the growth of Helianthus annuus. International Journal of Plant, Animal and Environmental Sciences, 2(4).

Khan M. A., Sharmaand V. and Shukla R. K. 2016. Response of sunflower (Helianthus annuus L.) to organic manure and biofertilizer under different levels of mycorrhiza and sulphur in comparison with inorganic fertilizer. Journal of Crop and Weed. 12(1):81-86. Kumar, J. and Badiyala, D. (2005). Effect of seed rate, row spacing and sowing time on yield attribute of soybean. Legume Res., 28:288-290.

Sattar, A., Kumar, M., Kumar, P. V. and Khan, S.A. (2017). Crop weather relation in kharif rice for north west alluvial plain zone of Bihar. J. Agrometeorol., 19:71-74.

\section{How to cite this article:}

Londhe, V. M., S. G. Birajdar, V. T. Jadhav, J. D. Jadhav and Amrutsagar, V. M. 2020. Impact of Weather Parameters on Yield of Kharif Sunflower (Helianthus annuus L.) under Different Growing Environments in Scarcity Zone of Maharashtra. Int.J.Curr.Microbiol.App.Sci. 9(09): 1396-1407. doi: https://doi.org/10.20546/ijcmas.2020.909.177 